\title{
Deep vein thrombosis caused by malignant afferent loop obstruction
}

\author{
Eun Gyu Kang ${ }^{1^{*}}$, Chan $\mathrm{Kim}^{2^{*}}$, Jeungeun Lee ${ }^{2}$, Min-uk Cha ${ }^{2}$, Joo Hoon Kim², Seo-Hwa Park', \\ Man Deuk $\mathrm{Kim}^{3}$, Do Yun Lee ${ }^{3}$, Sun Young $\mathrm{Rha}^{2}$ \\ ${ }^{I}$ Department of Internal Medicine, Hongik Hospital; ${ }^{2}$ Division of Medical Oncology, Department of Internal Medicine, \\ ${ }^{3}$ Department of Radiology, Yonsei University College of Medicine, Seoul, Korea
}

\begin{abstract}
Afferent loop obstruction following gastrectomy is a rare but fatal complication. Clinical features of afferent loop obstruction are mainly gastrointestinal symptoms. A 56-year-old female underwent radical total gastrectomy with Roux-en-Y esophagojejunostomy for treatment of advanced gastric cancer. After fourteen months postoperatively, she showed gradual development of edema of both legs. Computed tomography (CT) scan showed disease progression at the jejunojejunostomy site and consequent dilated afferent loop, which resulted in inferior vena cava (IVC) compression. A drainage catheter was placed percutaneously into the afferent loop through the intrahepatic duct and an IVC filter was placed at the suprarenal IVC, and selfexpanding metal stents were inserted into bilateral common iliac veins. With these procedures, sympotms related with afferent loop obstruction and deep vein thrombosis were improved dramatically. The follow-up abdominal CT scan was taken 3 weeks later and revealed the completely decompressed afferent loop and improved IVC patency. Surgical treatment should be considered as the first choice for afferent loop obstruction; however, because it is more immediate and less invasive, non-surgical modalities, such as percutaneous catheter drainage or stent placement, can be effective alternatives for inoperable cases or risky patients who have severe medical comorbidities.
\end{abstract}

Keywords: Afferent loop obstruction; Stomach cancer; Venous thrombosis; Interventional radiography

\section{INTRODUCTION}

Afferent loop obstruction is a rare and lethal complication of Billroth II gastrectomy or Roux-en-Y reconstitution following distal gastrectomy [1-3]. Afferent loop obstruction initially presents mainly as gastrointestinal (GI) symptoms, and the severity depends on the degree and duration of obstruction [6]. Afferent loop obstruction caused by complete obs-

Received: June 19, 2015, Revised: August 10, 2015

Accepted: August 12, 2015

Corresponding Author: Sun Young Rha, Department of Internal Medicine, Yonsei University College of Medicine,

50 Yonsei-ro, Seodaemun-Ku, Seoul 03722, Korea

Tel: +82-2-2228-8050, Fax: +82-2-362-5592

E-mail: rha7655@yuhs.ac

*Both authors contributed equally truction of the afferent limb could lead to persistent epigastric pain and vomiting. In this acute condition, high intraluminal pressure can lead to ischemia and perforation of the bowel wall of the afferent loop, ultimately resulting in serious peritonitis. When there is the partial obstrcution of the afferent limb, chronic symptoms such as intermittent postprandial pain can develop with projectile bilious vomiting. Once the diagnosis of afferent loop obstruction is made, prompt surgical intervention is required to reduce the high mortality rate associated with afferent loop obstruction and to minimize complications $[3,6,7]$.

We recently encountered a patient with afferent loop obstruction who initially presented with lower extremity edema rather than GI symptoms. In this patient, the severely distended afferent loop compressed the inferior vena cava (IVC),

Copyright (C) 2016 Yeungnam University College of Medicine

This is an Open Access article distributed under the terms of the Creative Commons Attribution Non-Commercial License (http://creativecommons.org/licenses/by-nc/4.0/) which permits unrestricted non-commercial use, distribution, and reproduction in any medium, provided the original work is properly cited. 
consequently resulting in deep vein thrombosis (DVT) below the level of compression. Invasive surgery was not an option due to the patient's poor general condition, thus non-surgical interventions were performed successfully without complications.

\section{CASE}

The 56-year-old female had undergone a radical total gastrectomy with Roux-en-Y esophagojejunostomy for stage IIIc automatic gain control (AGC) and subsequently received adjuvant chemotherapy with TS-1. Thirteen months postoperatively, she showed gradual development of nausea and vomiting. Upper endoscopy showed anastomosis site stenosis. Therefore, endoscopic balloon dilatation was performed and a self-expanding metallic stent was inserted. Abdominal computed tomography (CT) scan showed recurrence of AGC at the jejunal loop, extending to the incision site of the abdominal wall, while there was no evidence of afferent loop obstruction (Fig. 1A). Afterwards, she received palliative chemotherapy with 5-fluorouracil and docetaxel for 7 months. However, because the disease had progressed with tumor ingrowth into the stent, another stent was inserted and the chemotherapy regimen was changed to 5 -fluorouracil and oxaliplatin. However, after 2 months, edema of both legs became gradually aggravated; therefore she visited the emergency department under suspicion of DVT. On the initial laboratory finding, hemoglobin
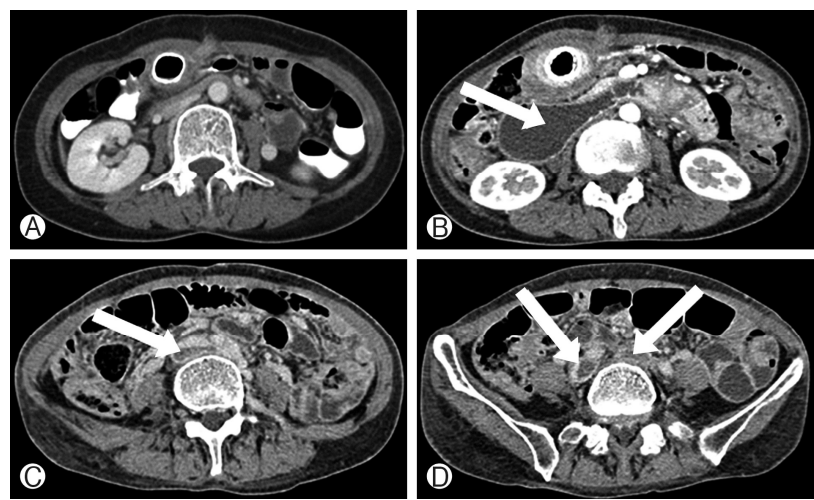

Fig. 1. Computed tomography (CT) scan of abdomen. (A) CT scan taken at the time of initial tumor recurrence. There was no obvious afferent loop obstruction. (B-D) CT scan taken at the time of both leg edema (10 months after initial recurrence). Tumor mass induced a distended afferent loop (B, arrow), resulting in thrombosis in the infrarenal inferior vena cava $(\mathrm{C}$, arrow) and bilateral common iliac veins ( $\mathrm{D}$, arrows). was $9.0 \mathrm{dL} / \mathrm{mg}$ and other laboratory results reflecting liver, kidney function were within normal range. Lower extremity CT scan taken on admission showed disease progression at the anastomosis site and consequently dilated afferent loop, which resulted in IVC compression with DVT involving infrarenal IVC and downstream veins including both common iliac veins, left femoral veins, and popliteal veins (Figs. 1B-1D). Therefore, anticoagulation with dalteparin was initiated immediately and an IVC filter was placed at the suprarenal IVC. At the same time, a multi-side hole drainage catheter was placed percutaneously into the afferent loop through the intrahepatic duct (Fig. 2A). After decompression, lower extremity edema began to improve with $\sim 1,000 \mathrm{cc}$ of drainage every day through the catheter. However, because symptoms were not completely resolved, the patient underwent aspiration thrombectomy with heparin infusion, and self-expanding metal stents were inserted into bilateral common iliac veins (Fig. 2B). Finally, her symptoms showed dramatic improvement and the patient was discharged from the hospital. The follow-up abdominal CT scan taken 3 weeks later showed the completely
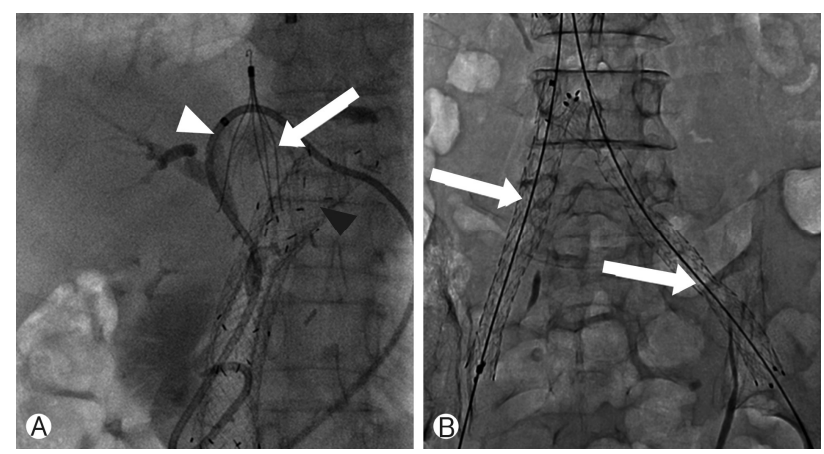

Fig. 2. Percutaneous interventions for treatment of afferent loop obstruction and deep vein thrombosis. (A) A multi-side hole drainage catheter (white arrowhead) was inserted percutaneously into the afferent loop through the common bile duct. At the same time, an inferior vena cava (IVC) filter (white arrow) was placed at the suprarenal IVC. Black arrowhead denotes a metallic stent which was previously inserted at the esophagojejunal anastomosis site. (B) Self-expanding metal stents (arrows) were placed percutaneously at the bilateral common iliac veins.

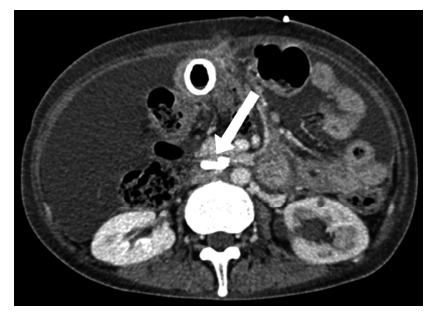

Fig. 3. Follow-up abdominal computed tomography scan taken 3 weeks later. The afferent loop was completely decompressed after percutaneous drainage. White arrow denotes the tip of the drainage catheter. 
decompressed afferent loop with much improved IVC compression and DVT, while disease had progressed with an increased amount of malignant ascites (Fig. 3). Since then, due to the patient's poor general condition, we were not able to proceed with further chemotherapy, and, after receiving 3 months of best supportive care, the patient expired.

\section{DISCUSSION}

Afferent loop obstruction is a rare but serious complication of Billroth II gastrectomy or Roux-en-Y procedure, which occurs in approximately $0.3-1.0 \%$ of cases [6,8,9]. In most cases, clinical presentations of afferent loop obstruction have been described as GI symptoms such as abdominal pain or vomiting, caused by increased intraluminal pressure and distension of the afferent limb [10], but, afferent loop obstruction sometimes presents with atypical symptoms. In the current patient, afferent loop obstruction initially presented as a DVT of IVC and downstream veins, which has not been previously reported. The largely distended afferent limb had a mass effect on IVC, thereby generating venous stasis and formation of a thrombus below the infrarenal IVC.

Diagnosis of afferent loop obstruction based on clinical symptoms is challenging, thus clinical suspicion of afferent loop obstruction in patients with previous history of gastrectomy is very important, and the possibility of afferent loop obstruction-induced mass effects should be considered.

Treatment of afferent loop obstruction can be categorized as surgical and non-surgical [12]. Although surgical conversion has been regarded as a standard treatment for afferent loop obstruction, in recent years, non-surgical procedures have been performed more frequently in patients who were not able to tolerate surgical procedures $[13,14]$. In particular, in most recent cases, afferent loop obstruction was caused by underlying malignancy and invasive surgical procedures were not an option due to patients' poor medical condition [12,14], therefore, an increasing number of patients with afferent loop obstruction will be candidates for non-surgical treatments.

There are two different routes for non-surgical treatment of afferent loop obstruction: the percutaneous and peroral routes [12]. The percutaneous route, which accesses the obstructed afferent limb through a percutaneous transhepatic catheter or enterostomy tube, is better for approaching the proximal or mid portion of the afferent loop [13]. Shortcomings of the percutaneous route include patients' inconvenience and increased risk of infection. Peroral routes use endoscopy for the placement of stents and are usually better for approaching distal obstruction at the anastomosis site [14]. While an endoscopically placed self-expanding metal stent minimizes patients' inconvenience and risk of infection, its shortcomings include stent migration and tumor ingrowth. Therefore, when choosing an appropriate treatment modality for afferent loop obstruction, clinicians should consider the pros and cons of each procedure and patients' underlying medical conditions

In conclusion, afferent loop obstruction can present as various clinical manifestations other than GI symptoms which should be included for differential diagnosis of patients with a previous surgical history of gastrectomy. Although surgical conversion is the treatment of choice, non-surgical interventions including percutaneous catheter drainage or stent placement can also be performed with immediate and excellent palliation in patients who are not amenable to surgery because of their medical comorbidities.

\section{REFERENCES}

1. Han K, Song HY, Kim JH, Park JH, Nam DH, Ryu MH, et al. Afferent loop syndrome: treatment by means of the placement of dual stents. Am J Roentgenol 2012;199:W761-6.

2. Jordan GL Jr. Surgical management of postgastrectomy problems. Arch Surg 1971;102:251-9.

3. Aoki M, Saka M, Morita S, Fukagawa T, Katai H. Afferent loop obstruction after distal gastrectomy with Roux-en-Y reconstruction. World J Surg 2010;34:2389-92.

4. Mitty Jr WF, Grossi C, Nealon Jr TF. Chronic afferent loop syndrome. Ann Surg 1970;172:996-1001.

5. Woodfield CA, Levine MS. The postoperative stomach. Eur J Radiol 2005;53:341-52.

6. Wise SW. Case 24: afferent loop syndrome 1. Radiology 2000;216:142-5.

7. Aimoto T, Uchida E, Nakamura Y, Katsuno A, Chou K, Tajiri $\mathrm{T}$, et al. Malignant afferent loop obstruction following pancreaticoduodenectomy: report of two cases. J Nippon Med Sch 2006;73:226-30.

8. Gayer G, Barsuk D, Hertz M, Apter S, Zissin R. CT diagnosis of afferent loop syndrome. Clin Radiol 2002;57:835-9.

9. Kim HC, Han J, Kim K, Kim Y, Yang HK, Kim S, et al. Afferent loop obstruction after gastric cancer surgery: helical CT findings. Abdom Imaging 2003;28:624-30.

10. Warrier RK, Steinheber FU. Afferent loop obstruction presenting as obstructive jaundice. Dig Dis Sci 1979;24:74-6.

11. Vettoretto N, Pettinato G, Romessis M, Bravo AF, Barozzi G, Giovanetti M. Laparoscopy in afferent loop obstruction presenting as acute pancreatitis. JSLS 2006;10:270-4.

12. Hosokawa I, Kato A, Shimizu H, Furukawa K, Miyazaki M. Percutaneous transhepatic metallic stent insertion for malig- 
nant afferent loop obstruction following pancreaticoduodenectomy: a case report. J Med Case Rep 2012;6:198.

13. Kim YH, Han JK, Lee KH, Kim TK, Kim KW, Choi BI. Palliative percutaneous tube enterostomy in afferent-loop syndrome presenting as jaundice: clinical effectiveness. J Vasc
Interv Radiol 2002;13:845-9.

14. Song HY, Kim TH, Choi EK, Kim JH, Kim KR, Shin JH, et al. Metallic stent placement in patients with recurrent cancer after gastrojejunostomy. J Vasc Interv Radiol 2007;18:1538-46. 